\title{
The importance of capillary density-stroke work mismatch for right ventricular adaptation to chronic pressure overload
}

\author{
Pierre-Emmanuel Noly, MD, MSc, ${ }^{\mathrm{a}, \mathrm{b}, \mathrm{c}}$ François Haddad, MD, ${ }^{\mathrm{b}, \mathrm{d}}$ Jennifer Arthur-Ataam, MSc, ${ }^{\text {,e }}$ \\ Nathaniel Langer, MD, ${ }^{\mathrm{a}, \mathrm{b}}$ Peter Dorfmüller, MD, PhD, ${ }^{\mathrm{a}, \mathrm{b}, \mathrm{e}, \mathrm{f}}$ Fanny Loisel, MSc, ${ }^{\mathrm{a}, \mathrm{b}}$ \\ Julien Guihaire, MD, MSc, ${ }^{\mathrm{b}, \mathrm{e}}$ Benoit Decante, MSc, ${ }^{\mathrm{b}}$ Lilia Lamrani, MSc, ${ }^{\mathrm{b}, \mathrm{c}}$ Elie Fadel, MD, PhD, ${ }^{\mathrm{a}, \mathrm{b}, \mathrm{e}, \mathrm{e}}$ and \\ Olaf Mercier, MD, $\mathrm{PhD}^{\mathrm{a}, \mathrm{b}, \mathrm{c}, \mathrm{e}}$
}

\section{ABSTRACT}

Objective: Mechanisms of right ventricular (RV) adaptation to chronic pressure overload are not well understood. We hypothesized that a lower capillary density (CD) to stroke work ratio would be associated with more fibrosis and RV maladaptive remodeling.

Methods: We induced RV chronic pressure overload over a 20-week period in 2 piglet models of pulmonary hypertension; that is, a shunt model $(n=5)$ and a chronic thromboembolic pulmonary hypertension model $(n=5)$. We assessed hemodynamic parameters and RV remodeling as well as RV CD, fibrosis, and angiogenic factors expression.

Results: Although RV was similarly hypertrophied in both models, maladapted RV remodeling with impaired systolic function was only seen in chronic thromboembolic pulmonary hypertension group members who had lower CD $\left(484 \pm 99\right.$ vs $\left.1213 \pm 74 \mathrm{cap} / \mathrm{mm}^{2} ; P<.01\right)$, lower CD to stroke work ratio $(0.29 \pm 0.07$ vs $0.82 \pm 0.16 ; P=.02)$, higher myocardial fibrosis $(15.4 \% \pm 3.8 \%$ vs $8.0 \% \pm 2.5 \% ; P<.01)$, as well as a higher angiogenic and fibrosis factors expression.

Conclusions: The RV adaptive response to chronic pressure overload differs between 2 different piglet models of PH. Mismatch between angiogenesis and workload (CD to stroke work ratio) was associated with greater degree of myocardial fibrosis and RV dysfunction and could be a promising index of RV maladaptation. Further studies are needed to understand the underlying mechanisms. (J Thorac Cardiovasc Surg 2017;154:2070-9)

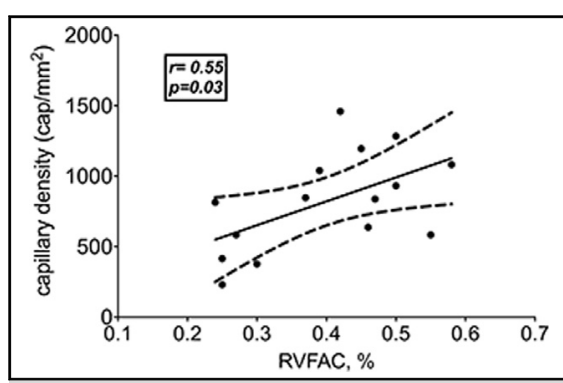

Correlation between capillary density, fibrosis, and right ventricular function in 2 models of right ventricle pressure overload.

\section{Central Message}

Mismatch angiogenesis/workload was associated with greater degree of right ventricular dysfunction in animal models. The capillary density to stroke work ratio could be a promising index to assess right ventricular adaptation to pressure overload.

\section{Perspective}

Discrepancy between the capillary density and the right ventricular stroke work may help us to understand the inability of end-stage right ventricular pulmonary hypertension to use oxygen correctly despite the metabolic shift. A major clinical implication of our study is that it offers, in a large animal model, support for therapies targeting neovascularization or angiogenesis.

See Editorial Commentary page 2080.
In the modern era, pulmonary hypertension $(\mathrm{PH})$ remains a progressive and often fatal disease despite the use of targeted therapies. ${ }^{1}$ Many studies have shown that right ventricular adaptation to pressure overload is the main

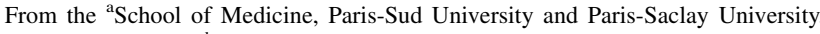
Kremlin-Bicêtre; ${ }^{\mathrm{b}}$ Research and Innovation Unit, ${ }^{\mathrm{c}}$ Department of Thoracic and Vascular Surgery and Heart-Lung Transplantation, DHU Thorax Innovation, INSERM UMR-S 999, LabEx LERMIT, and ${ }^{\mathrm{f}}$ Department of Pathology, Marie Lannelongue Hospital, Le Plessis Robinson, France; and ${ }^{\mathrm{d}}$ Division of Cardiovascular Medicine, Stanford University, Palo Alto, Calif.

P.-E.N. and F.H. contributed equally to this article. determinant of survival in patients with chronic pressure overload state (CPOS). ${ }^{2-5}$ Some etiologies in $\mathrm{PH}$ and CPOS are known to be associated with better right ventricular adaptation and survival. For example, a

Received for publication Oct 30, 2016; revisions received May 21, 2017; accepted for publication May 31, 2017; available ahead of print July 13, 2017.

Address for reprints: Olaf Mercier, MD, PhD, Department of Thoracic and Vascular Surgery and Heart-Lung Transplantation, Marie Lannelongue Hospital, 133 ave de la Résistance, 92350 Le Plessis Robinson, France (E-mail: o.mercier@ccml.fr). $0022-5223 / \$ 36.00$

Copyright (C) 2017 by The American Association for Thoracic Surgery http://dx.doi.org/10.1016/j.jtcvs.2017.05.102 


\begin{tabular}{|c|c|}
\hline \multicolumn{2}{|c|}{ Abbreviations and Acronyms } \\
\hline $\mathrm{CD}$ & $=$ capillary density \\
\hline $\mathrm{CI}$ & $=$ cardiac index \\
\hline CPOS & $=$ chronic pressure overload states \\
\hline CTEPH & $\begin{aligned}= & \text { chronic thromboembolic } \\
& \text { pulmonary hypertension }\end{aligned}$ \\
\hline $\mathrm{Ea}$ & $=$ pulmonary vascular elastance \\
\hline Ees & $=$ ventricular elastance \\
\hline ES & $=$ Eisenmenger syndrome \\
\hline mPAP & $=$ mean pulmonary arterial pressure \\
\hline PA & $=$ pulmonary artery \\
\hline $\mathrm{PH}$ & $=$ pulmonary hypertension \\
\hline RVFAC & $\begin{aligned}= & \text { right ventricular fractional area } \\
& \text { change }\end{aligned}$ \\
\hline SW & $=$ stroke work \\
\hline VEGF & $=$ vascular endothelium growth factor \\
\hline VEGF- & $\begin{aligned}= & \text { vascular endothelium growth factor } \\
& \text { type } \mathrm{A}\end{aligned}$ \\
\hline
\end{tabular}

Scanning this $Q R$ code will take you to a supplemental video for this article.

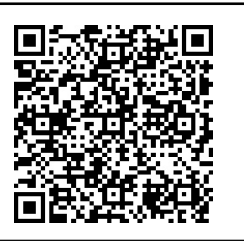

recent prospective multicenter study has shown that the survival of patients with idiopathic, postembolic, or anorexigen-induced $\mathrm{PH}$ is $58 \%$ at 3-year follow-up ${ }^{1}$ compared with $58 \%$ at 10-year follow-up for patients with congenital heart disease-associated $\mathrm{PH}$ and Eisenmenger syndrome (ES). ${ }^{6}$ In these patients, the main cause of death remains right ventricular failure $(36 \%){ }^{1}$

Although adaptive remodeling is often contrasted with maladaptive remodeling, the mechanisms underlying this transition are not well understood. Preclinical animal models suggested that a mismatch between right ventricular hypertrophy and right ventricular angiogenesis as well as altered metabolism could induce myocardial ischemia and promote the development of right heart failure. ${ }^{7-11} \mathrm{We}$ hypothesize that a mismatch between hypertrophy and angiogenesis, and workload of the right ventricle could be associated with an impairment of right ventricular function. However, limited data are available from studies comparing right ventricular capillary density (CD) of ES and non-ES patients. In this context, we performed an experimental study using 2 different models of chronic right ventricular pressure overload in piglets. In the first model, the increase in pulmonary artery (PA) pressure was obtained using a systemic-to-pulmonary shunt (Blalock-Taussig procedure). ${ }^{12}$ In the second model, we performed a ligation of the left PA followed by 5 embolizations of the right lower PA to increase PA pressure ${ }^{13,14}$ (chronic thromboembolic pulmonary hypertension [CTEPH] model). We aimed to study the right ventricle in these 2 different pig models of CPOS to determine whether mismatch between angiogenesis and workload would be associated with a greater degree of myocardial fibrosis and right ventricular dysfunction.

\section{METHODS \\ Experimental Study}

Fifteen large white piglets were randomly allocated to 3 groups (Figure 1). All animals received humane care in compliance with the Principles of Laboratory Animal Care formulated by the National Society for Medical Research and the Guide for the Care and Use of Laboratory Animals prepared by the Institute of Laboratory Animal Resources and published by the National Institutes of Health (publication No. 86-23, revised 1996) and the guidelines from directive 2010/63/EU of the European Parliament on the protection of animals used for scientific purposes. All animals were studied at baseline and at 20-weeks' follow-up.

Approval for the study was granted by our institutional ethics review board. All animal experiments were performed under general anesthesia with the following protocol. After prophylactic cefotaxime and premedication with ketamine, induction of anesthesia was performed with $1 \%$ propofol $(3 \mathrm{mg} / \mathrm{kg})$ and cisatracurium allowing endotracheal intubation. General anesthesia was maintained with isoflurane in $21 \%$ oxygen (Clarys 2000; TAEMA, Air Liquid, Paris, France) during surgery as well as during hemodynamic and echographic assessments. At the end of the experiment, anesthesia and paralysis were interrupted and the animals were weaned from mechanical ventilation. Postoperative analgesia was provided with subcutaneous nalbuphine as required for 4 days. Animals were killed at the end of the last experiment by administration of a lethal dose of sodium thiopental followed by exsanguination.

Induction of CPOS. CPOS was induced either by a systemic-topulmonary shunt ${ }^{12}$ (shunt group, $\mathrm{n}=5$ ) or by a progressive obstruction of the pulmonary vascular bed (CTEPH group, $n=5$ ). These 2 CPOS groups were compared with sham-operated animals (sham group, $\mathrm{n}=5$ ). Shunt model. In shunted animals, a modified Blalock-Taussig procedure was performed by left inominate artery to pulmonary trunk transposition mimicking a patent ductus arteriosus. Through a left small thoracotomy, the left inominate artery was dissected and harvested after systemic heparinization (intravenous heparin sulfate $3000 \mathrm{UI}$ ). Using this vascular autograft $(10 \mathrm{~mm}$ diameter), we performed a bypass between the thoracic aorta (usually the aortic arch) and the pulmonary trunk. The patency of the shunt was assessed by Doppler ultrasound intraoperatively, and by auscultation (thrill) or by echocardiography during the postoperative period. This procedure achieves an extracardiac systemic-to-pulmonary shunt without right ventricular volume overload, because the pulmonary valve is competent. The overflow in the pulmonary vascular bed induces remodeling of the small PAs and led to an increase of pulmonary pressures and resistances after 12 weeks. ${ }^{15}$

CTEPH model. The CTEPH model is described elsewhere. ${ }^{13,14}$ Briefly, CPOS was induced after ligation of the left PA and weekly embolization of the right lower lobe PA with n-butyl-2-cyanoacrylate (Histoacryl; B. Braun Medical S.A., Diegem, Belgium) during 5 weeks to progressively occlude the pulmonary vascular bed. Pulmonary pressures increase progressively and the model is mature after 6 to 7 weeks of evolution.

Right heart hemodynamic assessment. Mean systemic blood pressure, heart rate, and peripheral oxygen saturation were monitored during the procedures. In addition, right heart catheterization was performed percutaneously via the right internal jugular vein using a Swan- 


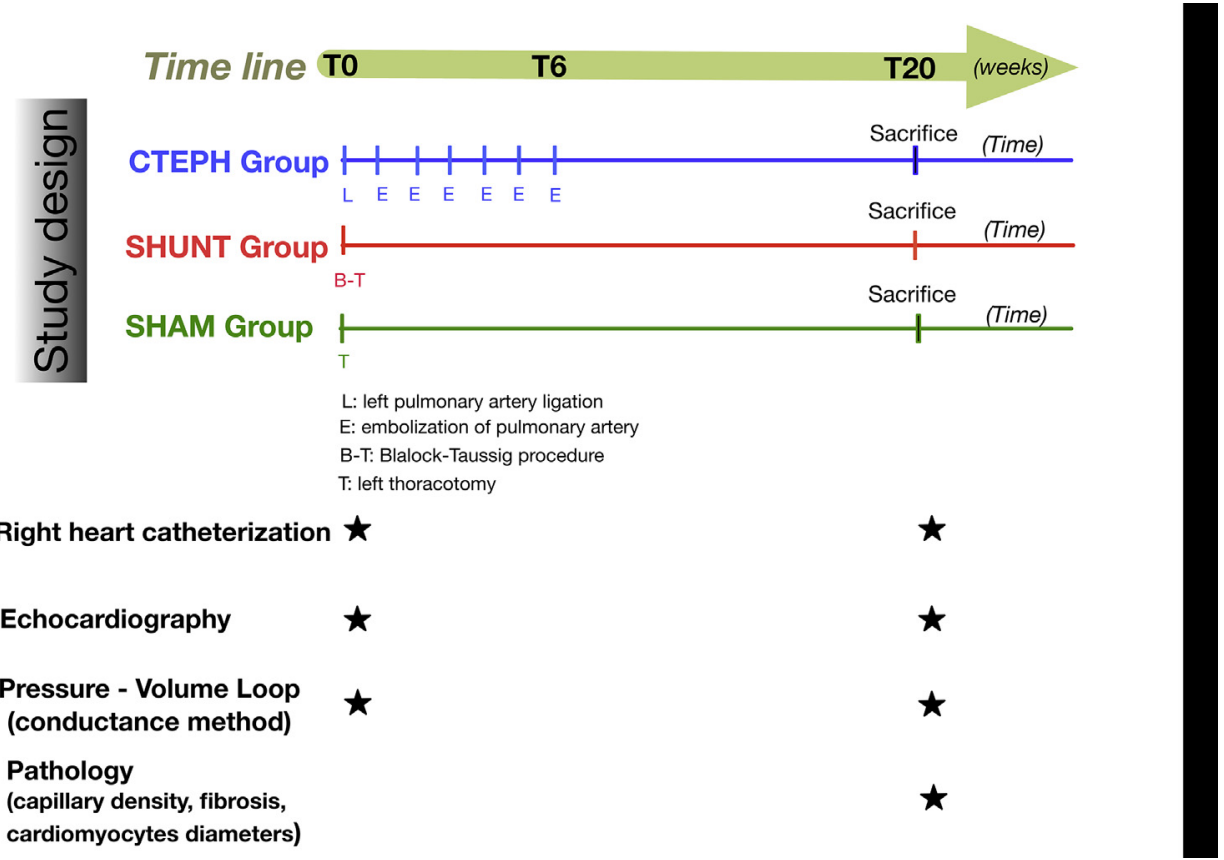

FIGURE 1. Study design. CTEPH, Chronic thromboembolic pulmonary hypertension.

Ganz probe. Mean PA pressure (mPAP), pulmonary capillary wedge pressure, central venous pressure, cardiac output, and mixed venous saturation were recorded. Body surface area, cardiac index, and total pulmonary vascular resistances (mPAP/cardiac output) were calculated. Because the aortopulmonary shunt may interfere with the thermodilution method, pulmonary hemodynamic parameters have been measured after shunt closure (percutaneous balloon inflation). In the shunt group animals, the ratio of the systemic-to-pulmonary flow was determined with the Fick method as previously described. ${ }^{16-18}$ Pressure-volume loops of the right ventricle were assessed by the conductance catheter technique. ${ }^{19}$ Right ventricular elastance (Ees) and right ventricular stroke work (SW) were recorded and pulmonary arterial elastance $(\mathrm{Ea})(\mathrm{Ea}=\mathrm{mPAP} /$ right ventricle stroke volume $)$ was calculated. Right ventricle-PA coupling was calculated as the ratio of Ees to Ea to assess right ventricular adaptation to pressure overload. ${ }^{20}$

Echocardiography. Transthoracic echocardiography was performed with a Vivid E9 General Electric System echograph (General Electric Company, Fairfield, Conn) at baseline and after 20 weeks. For all animals, we measured the right ventricular basal diastolic diameter, the right ventricular diastolic and systolic area, the end-diastolic right ventricle free wall thickness, the right ventricular fractional area change (RVFAC), the tricuspid annular plane systolic excursion, and the right ventricular myocardial performance index. ${ }^{21,22}$

Histology and immunohistochemistry. Histologic study. After hemodynamic and echocardiographic assessments, animals were killed and heart-lung blocs were harvested after an ice-cold hyperkalemic crystalloid cardioplegia. The Fulton ratio (right ventricle weight/[left ventricular + septum weight]) was calculated.

Tissue samples from the right ventricle were fixed overnight in $4 \%$ formalin and embedded in paraffin and 3- $\mu \mathrm{m}$ sections were stained with hematoxylin-eosin and red sirius stain. Slides were examined at $\times 400$ total magnification and analyzed by 2 independent investigators. Right ventricular myocardial hypertrophy was assessed by measuring the short axis diameter of 40 randomly chosen cardiomyocytes per field with 10 fields per animal.

$C D$ assessment. After deparaffinization and heat-induced epitope retrieval, myocardial samples were incubated with an antihuman Von
Willebrand factor antibody (Dako M 0082, rabbit antihuman; Dako, Santa Barbara, Calif). A goat antirabbit antibody combined with Alexa fluor 488 (Molecular probes; Fisher Invitrogen, Illkrich, France) was used to reveal the primary antibody. The $\mathrm{CD}$ of the right ventricle was determined by the number of capillaries per millimeter ${ }^{2}$ in at least 20 microscopic fields examined at $\times 400$ total magnifications.

Fibrosis assessment. Right ventricular myocardial fibrosis was automatically quantified using ImageJ version 1.4 software (available at: http://www.imagej.net) from 10 fields per red sirius staining slide. As previously described, we calculated the ratio between fibrosis and the total amount of tissue assessed by determining the total tissue areas occupied by cardiomyocytes and collagen, excluding the lumen (ie, empty spaces), as well as excluding annotations of perivascular fibrosis. ${ }^{23}$

Real-time quantification by polymerase chain reaction. Total RNA extraction was performed using the Qiagen Tissue Lyser (Qiagen, Hilden, Germany) and the phenol-chloroform method. Reverse transcription was performed using $1 \mu \mathrm{g}$ RNA with the Quantitect Reverse Transcription Kit (Qiagen) according to the manufacturer's instructions. Expression of vascular endothelium growth factor (VEGF) type a (VEGF-A), VEGF receptor type 1 and VEFG receptor type 2 (VEGFR-2) were analyzed with specific TaqMan (Applied Biosystems, Foster City, Calif) probe VEGF-A (Ss: 03393993_m1), VEGFR receptor type 1 (Ss: 03375679_u1), VEGR receptor type 2 (Ss: 03375683_1), hypoxia-inducible factor $1 \alpha$ (HIF-1 $\alpha$ ) (Ss: 03390447_m1), and tissue growth factor type $\beta$ (Ss: 03382325_u1). Relative quantification was achieved with the comparative $2^{-\Delta \Delta \mathrm{Ct}}$ method by normalization with the 18 S RNA.

Western blot. Protein extracts (100 $\mu \mathrm{g}$ ) were resolved on $4 \%$ to $15 \%$ sodium dodecyl sulfate polyacrylamide gel and electrotransferred to nitrocellulose membranes (Biorad; Marnes la Coquette, France). After blocking with $5 \%$ body surface area in $0.1 \%$ polysorbate-tris buffered saline (tris-hydrochloric acid [pH 8.0] and sodium chloride $150 \mathrm{mM}$ ), membranes were incubated with anti-Hif- $1 \alpha$ monoclonal antibody (1:250; purified mouse antihuman Hif- $1 \alpha$, NS 610959; BD Bioscience, Franklin Lakes, NJ) and rabbit anti-VEGF-A polyclonal antibody (1:250; 
TABLE 1. Physiologic data of the 2 groups of chronic pressure overload state (chronic thromboembolic pulmonary hypertension [CTEPH] and shunt) compared with the sham group at 20 weeks

\begin{tabular}{|c|c|c|c|}
\hline Variable & CTEPH $(\mathbf{n}=\mathbf{5})$ & Shunt $(\mathbf{n}=\mathbf{5})$ & Sham $(n=5)$ \\
\hline \multicolumn{4}{|l|}{ Morphology } \\
\hline Weight $(\mathrm{kg})$ & $52.2 \pm 5.0$ & $49.7 \pm 5.0$ & $43.2 \pm 3.3$ \\
\hline Body area $\left(\mathrm{m}^{2}\right)$ & $1.24 \pm 0.08$ & $1.20 \pm 0.08$ & $1.08 \pm 0.05$ \\
\hline \multicolumn{4}{|l|}{ Hemodynamic parameters } \\
\hline HR (bpm) & $86.6 \pm 7.5$ & $91.8 \pm 5.3$ & $89.4 \pm 6.4$ \\
\hline Oxygen saturation $(\%)$ & $96.6 \pm 1.3$ & $97.6 \pm 2.0$ & $99.2 \pm 0.9$ \\
\hline Systemic arterial $\mathrm{PaO}_{2}(\mathrm{~mm} \mathrm{Hg})$ & $72.48 \pm 6.91$ & $84.64 \pm 4.12$ & $81.48 \pm 1.79$ \\
\hline $\mathrm{SvO}_{2}(\%)$ & $59.8 \pm 5.9^{*}$ & $71.50 \pm 3.7$ & $79.4 \pm 2.8$ \\
\hline $\mathrm{mAP}(\mathrm{mm} \mathrm{Hg})$ & $84.0 \pm 11.1$ & $76.2 \pm 3.0$ & $75.6 \pm 7.9$ \\
\hline $\mathrm{mPAP}(\mathrm{mm} \mathrm{Hg})$ & $26.8 \pm 1.4^{*}$ & $22.8 \pm 1.0^{\dagger}$ & $14.8 \pm 0.8$ \\
\hline $\mathrm{mPAP} / \mathrm{mAP}$ & $0.33 \pm 0.04^{*}$ & $0.29 \pm 0.01$ & $0.19 \pm 0.02$ \\
\hline $\mathrm{RAP}(\mathrm{mm} \mathrm{Hg})$ & $6.8 \pm 0.6^{*}$ & $7.2 \pm 0.8 \dagger$ & $4.8 \pm 0.9$ \\
\hline $\mathrm{LAP}(\mathrm{mm} \mathrm{Hg})$ & $8.60 \pm 0.5^{*}$ & $8.20 \pm 0.4 \dagger$ & $5.80 \pm 0.4$ \\
\hline $\mathrm{CO}(\mathrm{L} / \mathrm{min})$ & $3.9 \pm 0.3$ & $5.7 \pm 0.6+$ & $3.6 \pm 0.2$ \\
\hline $\mathrm{CI}\left(\mathrm{L} / \mathrm{min} / \mathrm{m}^{2}\right)$ & $3.3 \pm 0.5$ & $4.7 \pm 0.3 \dagger$ & $3.1 \pm 0.2$ \\
\hline Calculated RVSV (mL) & $46.4 \pm 3.6 \ddagger$ & $65.3 \pm 7.05 \dagger$ & $44.1 \pm 2.6$ \\
\hline TPVR (dynes $/ \mathrm{sec} / \mathrm{cm}^{5}$ ) & $553.6 \pm 48.0^{*} \ddagger$ & $287 \pm 49.5$ & $296.4 \pm 23.8$ \\
\hline \multicolumn{4}{|l|}{ Pressure-volume loops analysis } \\
\hline RVSW (mm Hg/min) & $2192 \pm 255.9^{*}$ & $2110 \pm 174.2 \dagger$ & $1124 \pm 44.06$ \\
\hline Ees $(\mathrm{mm} \mathrm{Hg} / \mathrm{min})$ & $0.40 \pm 0.12$ & $0.31 \pm 0.07$ & $0.28 \pm 0.06$ \\
\hline $\mathrm{Ea}(\mathrm{mm} \mathrm{Hg} / \mathrm{min})$ & $0.44 \pm 0.17^{*}$ & $0.23 \pm 0.04$ & $0.20 \pm 0.02$ \\
\hline Right ventricle-pulmonary artery coupling & $0.71 \pm 0.15^{*}$ & $1.35 \pm 0.32$ & $1.39 \pm 0.27$ \\
\hline \multicolumn{4}{|l|}{ Echocardiography } \\
\hline Indexed right ventricle diastolic area $\left(\mathrm{cm}^{2} / \mathrm{m}^{2}\right)$ & $9.7 \pm 0.6^{*}$ & $7.1 \pm 1.3$ & $5.2 \pm 0.2$ \\
\hline Indexed left ventricle diastolic area $\left(\mathrm{cm}^{2} / \mathrm{m}^{2}\right)$ & $20.3 \pm 3.6$ & $23.0 \pm 2.1$ & $16.1 \pm 1.9$ \\
\hline Right ventricle/left ventricle area & $0.55 \pm 0.1^{*} \ddagger$ & $0.30 \pm 0.05$ & $0.34 \pm 0.05$ \\
\hline Right ventricle basal diastolic diameter $(\mathrm{cm})$ & $10.72 \pm 1.36^{*}$ & $9.55 \pm 1.84$ & $6.72 \pm 1.14$ \\
\hline RVFAC \% & $0.26 \pm 0.01 * \ddagger$ & $0.46 \pm 0.03$ & $0.46 \pm 0.02$ \\
\hline $\operatorname{LVEF}(\%)$ & $52.2 \pm 6.1$ & $59.2 \pm 5.6$ & $52.2 \pm 12.5$ \\
\hline
\end{tabular}

Values are presented as mean \pm standard deviation. $\mathrm{CTEPH}$, Chronic thromboembolic pulmonary hypertension; $\mathrm{HR}$, heart rate; $\mathrm{PaO}_{2}$, arterial oxygen pressure; $\mathrm{SVO}_{2}$, venous saturation in oxygen; $m A P$, mean systemic arterial pressure; $m P A P$, mean pulmonary arterial pressure; $R A P$, right atrial pressure; $L A P$, left atrial pressure; $C O$, cardiac output; $C I$, cardiac index; $R V S V$, right ventricular stroke volume; $T P V R$, total pulmonary vascular resistance; $R V S W$, right ventricular stroke work; Ees, elastance of RV; Ea, elastance of the pulmonary artery; $R V F A C$, right ventricular fractional area change; $L V E F$, left ventricle ejection fraction. $* P<.05 \mathrm{CTEPH}$ versus sham. $\dagger P<.05$ shunt versus sham. $\ddagger P<.05$ shunt versus CTEPH.

VEGF 147: sc-507; Santa Cruz Biotechnology, Santa Cruz, Calif). After incubation with secondary horse antimouse antibody (1:5000) immunoreactive bands were detected using chemiluminescence with Biorad Kit in a Chemidoc (Biorad) machine. Relative quantification was performed by normalization with glyceraldehyde 3-phosphate dehydrogenase.

\section{Statistical Analysis}

The number of animals required was determined to obtain a difference of mPAP of $30 \%$ between models and sham. Eighteen piglets were necessary to obtain 5 animals in the 3 groups ( $16 \%$ of mortality). All data were expressed as mean values \pm standard deviation. For histology, average values for each parameter in each animal were calculated. The Kolmogorov-Smirnov test was applied to test for normality of the distribution of each variable. After 20 weeks, the 3 groups were compared by 1-way analysis of variance, including post hoc analysis by Bonferroni posttest adjustment. Relationships between $\mathrm{CD}$ or fibrosis and right ventricle-PA coupling or with noninvasive index of right ventricular function were approached using linear regression and Pearson correlation. Statistical analyses were performed using GraphPad Prism 6 (GraphPad Software, San Diego, Calif) and SPSS version 22.0 for Mac (IBM-SPSS Inc, Armonk, NY).

\section{RESULTS}

\section{Animal Study}

Fifteen 1-month-old large white male piglets were studied at baseline and after 20 weeks. At baseline, weight $(18.8 \pm 1.16 \mathrm{~kg})$, body surface area, and hemodynamic characteristics were not statistically different between groups (data not shown). 

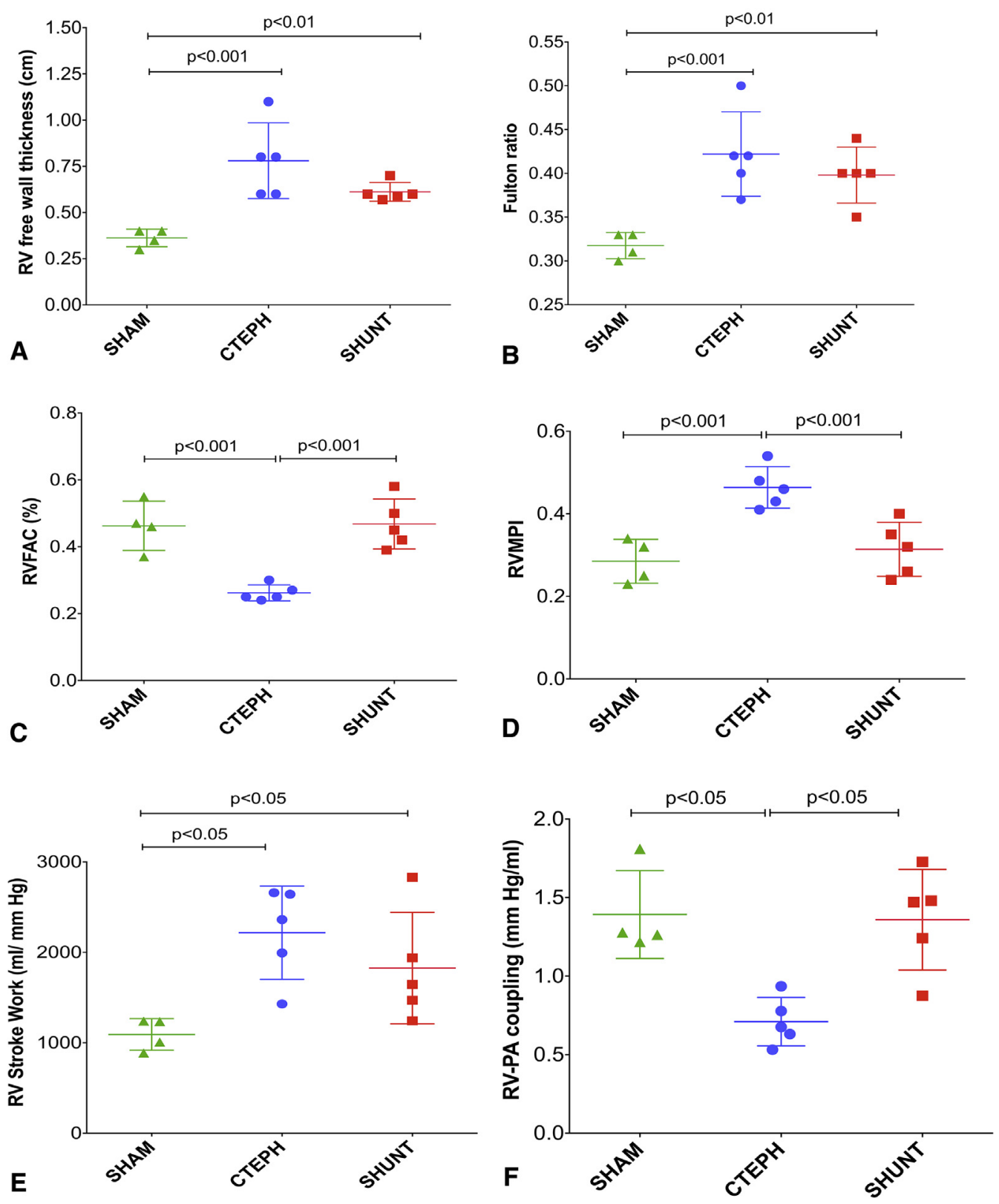

FIGURE 2. Right ventricular remodeling assessed by echocardiography, pressure-volume loops, and pathology. Pressure overload induced right ventricular hypertrophy in both chronic thromboembolic pulmonary hypertension $(C T E P H)$ and shunt groups. A, Right ventricular hypertrophy was confirmed by increased right ventricle $(R V)$ free wall thickness in echocardiography. B, Right ventricular hypertrophy was confirmed by increased Fulton ratio. C, Right ventricular systolic function was significantly impaired in the CTEPH group with a lower right ventricular fractional area change (RVFAC). D, Right ventricular systolic function was significantly impaired in the CTEPH group a higher right ventricular myocardial performance index (RVMPI). Pressure-volume loops study revealed an increased RV SW in both CTEPH and shunt groups, whereas the RV-pulmonary artery $(R V-P A)$ coupling was only impaired in the CTEPH group. E, Right ventricular SW was significantly increased in both CTEPH and SHUNT groups. F, Right ventricle-PA coupling is decreased only in the CTEPH group.

Hemodynamic assessment. Hemodynamic data are summarized in Table 1. Briefly, the CTEPH and shunt groups had both chronic pulmonary pressure overload with increase of mPAP and a decrease of the mPAP to mean arterial systemic pressure ratio compared with the sham group. Twenty weeks after induction of pressure overload, mPAP was significantly elevated in the shunt and CTEPH animals $(23 \pm 1 \mathrm{~mm} \mathrm{Hg}$ and $27 \pm 1 \mathrm{~mm} \mathrm{Hg}$, respectively) compared with sham animals $(15 \pm 1 \mathrm{~mm}$ $\mathrm{Hg} ; P<.01)$. Cardiac index and cardiac output were higher in the shunt group compared with sham and CTEPH animals. The systemic-to-pulmonary flow ratio (Fick 

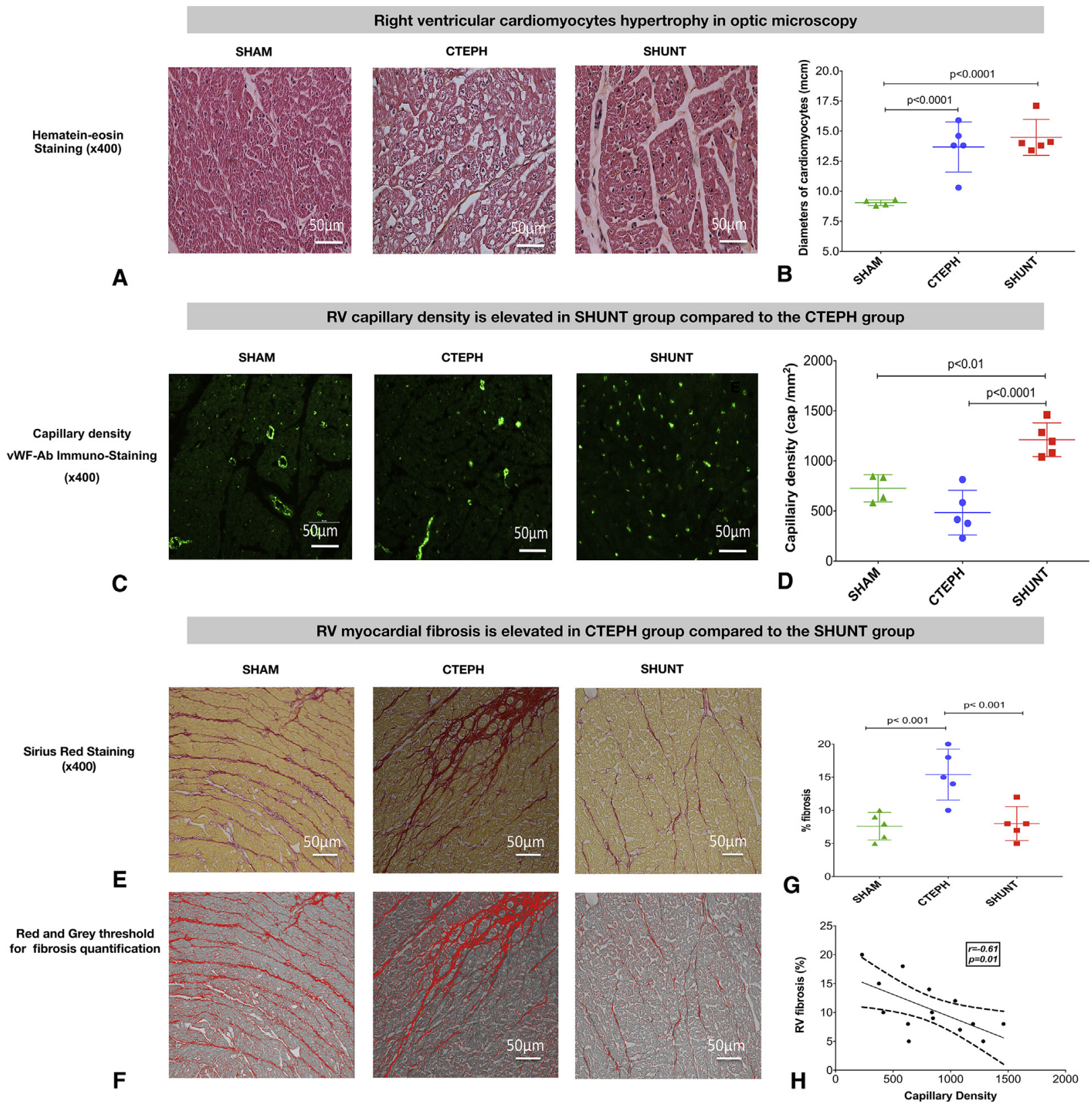

FIGURE 3. Cardiomyocytes size, capillary density, and fibrosis in the right ventricle (representative sections $\times 400$ magnification). A and B, Hematoxylineosin stained sections demonstrating that the transverse diameter of the cardiomyocytes was significantly higher in the chronic thromboembolic pulmonary hypertension $(C T E P H)$ and shunt groups compared with the sham group $(14.6 \pm 0.7 \mu \mathrm{m}$ and $14.1 \pm 0.5 \mu \mathrm{m}$ vs $9.6 \pm 0.3 \mu \mathrm{m} ; P=.0006)$. C and $\mathrm{D}, \mathrm{Capillary}$ density quantification was calculated based on immunostaining using Von Willebrand factor antibody. Capillary density was significantly higher in the shunt group compared with the CTEPH and sham groups $\left(1213 \pm 74 \mathrm{cap} / \mathrm{mm}^{2}\right.$ vs $484 \pm 99 \mathrm{cap} / \mathrm{mm}^{2}$ and $\left.657 \pm 116 \mathrm{cap} / \mathrm{mm}^{2} ; P<.01\right)$. E, Red sirius-stained sections showing that myocardial fibrosis rate automatically calculated with the described red threshold (F) was significantly higher in the CTEPH group compared with the shunt and the sham groups $(14.4 \% \pm 3.8 \%$ vs $8 \% \pm 2.5 \%$ and $8 \% \pm 1 \% ; P<.01)(\mathrm{G})$. H, Capillary density was inversely correlated to fibrosis rate. $R V$, Right ventricle.

method, pulmonary-systemic flow ratio $[\mathrm{Qp} / \mathrm{Qs}]$ ) was $1.66 \pm 0.25$ in the shunt group.

Structural remodeling of right ventricle to CPOSinduced right ventricular hypertrophy ratio in both PH models. After 20 weeks, the right ventricle was hypertrophied in both groups (Figure 2, $A$ and $B$ ).
Echocardiographic middle right ventricle free wall thickness was increased in shunt $(0.52 \pm 0.02 \mathrm{~cm})$ and CTEPH $(0.66 \pm 0.03 \mathrm{~cm})$ groups compared with sham animals $(0.41 \pm 0.02 \mathrm{~cm} ; P<.01)$. The Fulton ratio was also significantly elevated in shunt $(0.40 \pm 0.01)$ and CTEPH $(0.42 \pm 0.02)$ animals compared with sham animals 

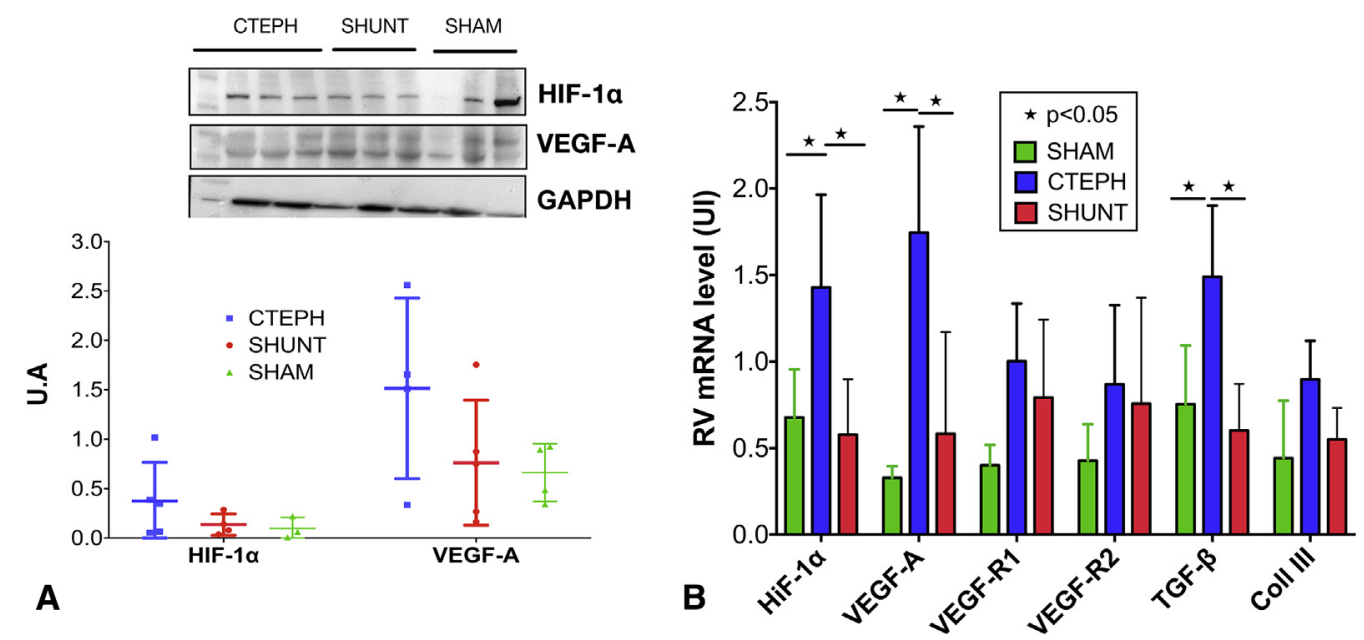

FIGURE 4. A, Hypoxia-inducible factor $1 \alpha(H I F-1 \alpha)$ and vascular endothelial growth factor type A $(V E G F-A)$ right ventricular protein levels as assessed by Western blot were higher in the chronic thromboembolic pulmonary hypertension $(C T E P H)$ group compared with the sham group $(P=.06)$. B, HIF- $1 \alpha$, VEGF-A, and tissue growth factor type $\beta(T G F-\beta)$ gene expressions were significantly higher in the CTEPH group compared with the shunt group $(P=.02$, $P=.02$, and $P=.01$, respectively). GAPDH, Glyceraldehyde-3-phosphate dehydrogenase; U.A., units arbitrary; $R V$, right ventricle; $U I$, units international; coll, collagen III.

$(0.32 \pm 0.01 ; P=.01)$. right ventricular hypertrophy was confirmed in optical microscopy in shunt and CTEPH animals, with an increase in transverse diameter of cardiomyocytes $(14.6 \pm 0.7 \mu \mathrm{m}$ and $14.1 \pm 0.5 \mu \mathrm{m})$ compared with sham animals $(9.6 \pm 0.3 \mu \mathrm{m} ; P<.01)$ (Figure 3, $A$ and $B$ ). According to echocardiograph findings, right ventricle enlargement was only found in the CTEPH group characterized by a higher indexed right ventricle end-diastolic area $\left(9.7 \pm 0.6 \mathrm{~cm}^{2}\right.$ vs $5.2 \pm 0.2 \mathrm{~cm}^{2}$ sham or $7.1 \pm 1.3$ shunt; $P=.03$ ) and a higher right ventricle to left ventricle area ratio (Table 1).

Functional remodeling of right ventricle: Right ventricular function was maladaptive in the CTEPH group and adaptive in the shunt group. According to echocardiography, right ventricular function was impaired in the CTEPH group and remained stable in the shunt group (Figure 2). Compared with shunt animals, CTEPH animals had a higher right ventricular myocardial performance index $(0.48 \pm 0.04$ vs $0.30 \pm 0.02 ; P=.02)$, a lower RVFAC $(25.0 \% \pm 1.0 \%$ vs $49.0 \% \pm 4.3 \% ; P<.01)$ (Figure $2, C$ and $D$ ), and a lower tricuspid annular plan systolic excursion $(1.11 \pm 0.07$ vs $1.5 \pm 0.09 \mathrm{~mm} ; P<.01)$. The right ventricle diastolic area and the basal diastolic diameter were increased in the CTEPH group, demonstrating right ventricle dilatation (Table 1).

Right ventricular SW was increased in both the CTEPH and the shunt groups compared with the sham group (Figure 2,E). However, CTEPH animals had a great degree of altered ventriculoarterial coupling $(\mathrm{Ea} / \mathrm{Ees} 0.71 \pm 0.15$ vs $1.35 \pm 0.32$ for shunt and $1.39 \pm 0.27$ for sham; $P=.02$ ) (Figure 2,F) despite elevation of the Ees (not statistically significant) compared with the shunt group (Table 1).
High CD, CD to SW ratio, and low myocardial fibrosis were associated with adaptive right ventricle remodeling. $C D$ was higher in the shunt group compared with the CTEPH group $\left(1213 \pm 74 \mathrm{cap} / \mathrm{mm}^{2}\right.$ vs $484 \pm 99 \mathrm{cap} / \mathrm{mm}^{2}$ and $657 \pm 116 \mathrm{cap} / \mathrm{mm}^{2}$ in the sham group; $P<.01$ ) (Figure 3,C and $D$ ). We found regularly distributed myocardial vessels in all groups. Conversely, diffuse right ventricular myocardial fibrosis was higher in the CTEPH group compared with the shunt group $(15.4 \% \pm 3.8 \%$ vs $8.0 \% \pm 2.5 \%$ and $8.0 \% \pm 1.0 \%$ in the sham group; $P<.01$ ) (Figure 3, $E$ and $G$ ). Right ventricular $C D$ and myocardial fibrosis were inversely correlated (Figure 3, $H$ ). Fibrosis factors (eg, tissue growth factor type $\beta$.) were overexpressed within the myocardium of CTEPH animals. This is consistent with the observed right ventricular myocardial fibrosis enhancement in this group (Figure 4). Despite a lower right ventricle CD in the CTEPH group, angiogenic factors (eg, VEGF and HIF-1 $\alpha$ ) were increased in the right ventricle of CTEPH animals (Figure 4).

CTEPH animals had the lowest myocardial CD to SW ratio, which is also correlated with right ventricle-PA coupling (Figure 5, $A$ and $B$ ). RVFAC and right ventricle-PA coupling were correlated to $C D$ and inversely correlated to myocardial fibrosis (Figure 5, $C-F$ ).

\section{DISCUSSION}

The main finding of our study is that CD-SW mismatch is strongly associated with myocardial fibrosis and altered right ventricle-PA coupling in CPOS. Although HIF- $1 \alpha$ and VEGF-A were upregulated in the presence of a lower a lower CD to SW ratio, this did not appear sufficient to compensate for the decreased CD (Video 1). 

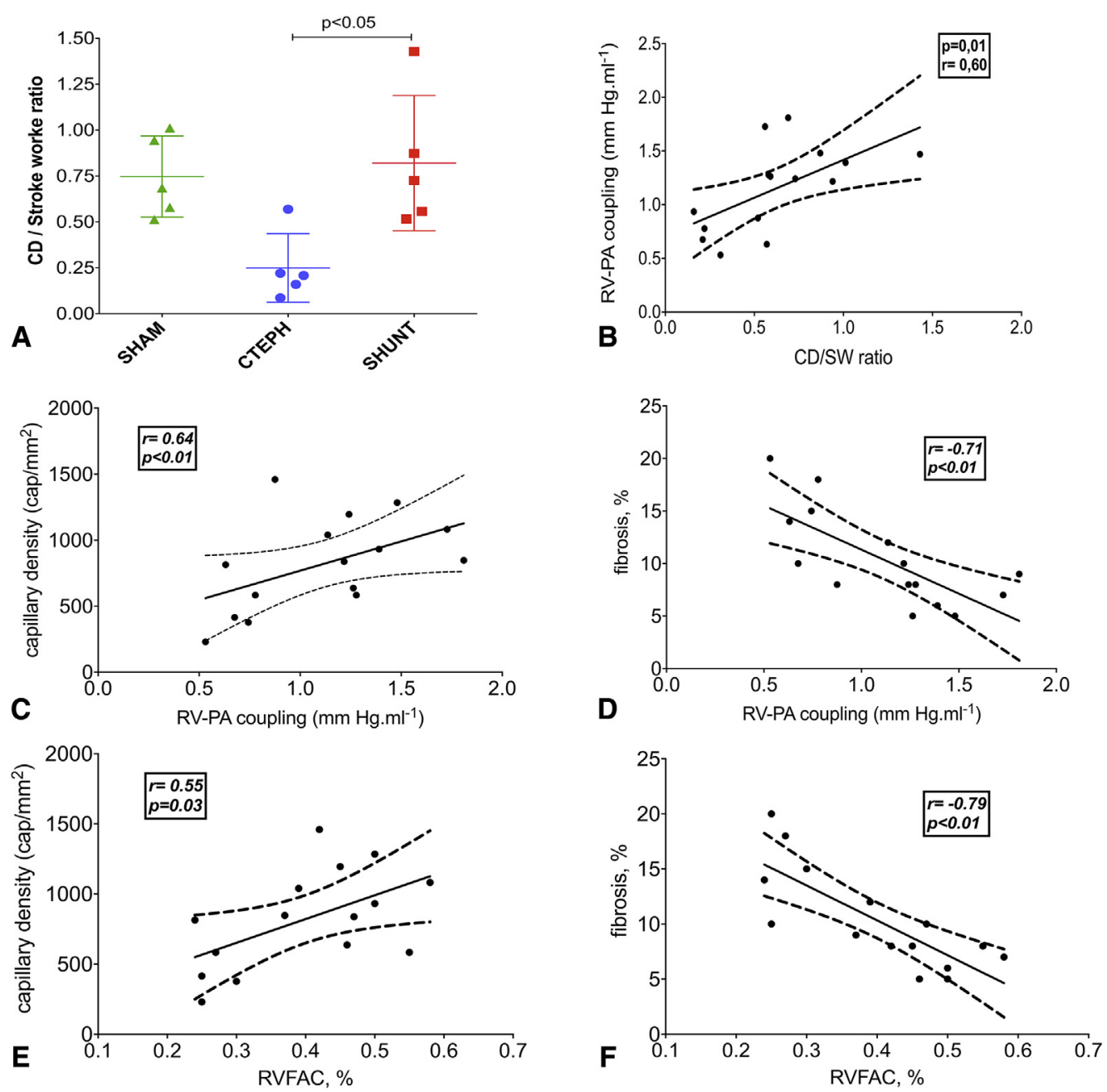

FIGURE 5. Correlations between capillary density, fibrosis, and right ventricular function. A, The capillary density-to-stroke work ratio $(C D / S W)$ was significantly lower in the chronic thromboembolic pulmonary hypertension $(C T E P H)$ group compared with the shunt group $(0.29 \pm 0.07$ vs $0.82 \pm 0.16$; $P=.02)$. B, CD/SW was also correlated with right ventricle-pulmonary artery $(R V-P A)$ coupling $(r=.60 ; P=.02)$. C and E, RV-PA coupling and right ventricular fractional area change $(R V F A C)$ were positively correlated with right ventricular CD. D and F, RV-PA coupling and RVFAC were negatively correlated with fibrosis. A mismatch between $\mathrm{CD}$ and right ventricular SW was associated with an impairment of the right ventricular systolic function and pulmonary artery coupling under chronic pulmonary pressure overload states. All statistical analyses were performed with $\mathrm{n}=5$ piglets per group.

One of the original contributions of our study is that it validates original observations made in small animal models. The models we developed reproduce the physiology of CPOS with maladaptative remodeling (CTEPH model) and CPOS with adaptative remodeling, although at a lower degree of total pulmonary vascular resistance (shunt model). Boogard and colleagues ${ }^{7}$ were the first to describe a decrease in $\mathrm{CD}$ in failing right ventricle compared with adapted right ventricle. They studied 2 different models of PH in rats: the PA banding model inducing adaptive right ventricular remodeling and hypoxia + VEGFR blocker (Sugen, SU5416) model inducing maladaptive right ventricle remodeling. The CD was correlated to right ventricular adaptation. However, these animal models did not replicate Eisenmenger physiology. Moreover, hypoxia + Sugen may interfere with myocardial angiogenesis. Our models did not use any antiangiogenic compound and showed different CD related to right ventricular adaptation. Our results confirmed those from Bogaard and colleagues ${ }^{7}$ in 2 different $\mathrm{PH}$ animal models in that $\mathrm{CD}$ correlates with right ventricular adaptation. More recently, Sutendra and colleagues $^{8}$ showed, in a monocrotaline rat model, that right ventricular $\mathrm{CD}$ was lower in decompensated right ventricular hypertrophy rather than in compensated right ventricular hypertrophy.

Although not proven by this study, the CD-SW mismatch may contribute to myocardial perfusion defects and ischemia in a chronically overloaded right heart. Right ventricular ischemia is a recognized cause of the transition from adaptive to maladaptive right ventricular remodeling. ${ }^{8,24}$ Baldwa and colleagues ${ }^{25}$ demonstrated lower right 


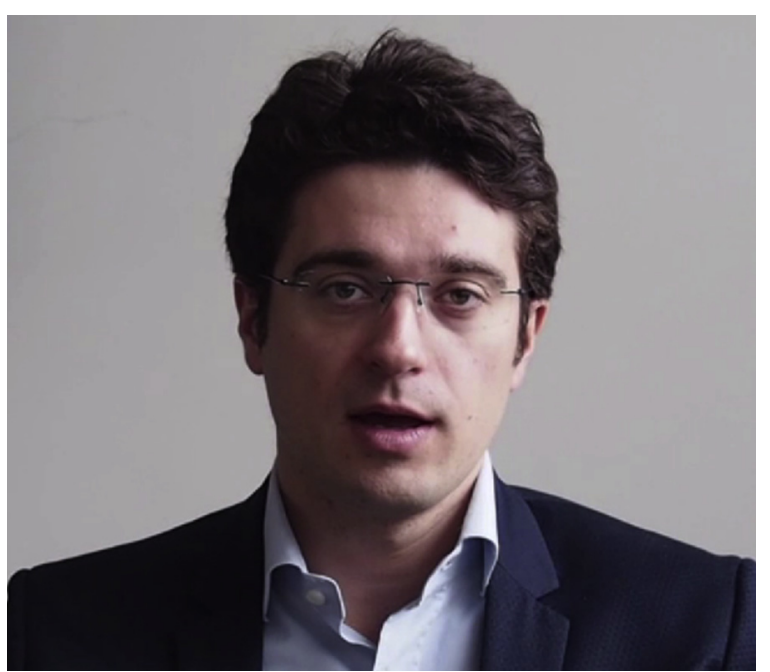

VIDEO 1. Dr Pierre-Emmanuel Noly explains the main findings of this study and its importance in the field of pulmonary hypertension disease. Video available at: http://www.jtcvsonline.org/article/S0022-5223(17) 31183-2/fulltext.

ventricular perfusion on cardiac perfusion scan with increased troponin blood level in patients with severe PH. In addition, high-pressure overload induces high right ventricle pressure during systole, preventing the usual systole-diastolic right ventricular coronary artery perfusion. ${ }^{26}$ Relationships between right ventricular angiogenesis and hypertrophy pathways have been studied over the past few years. ${ }^{27-30}$ Sano and colleagues ${ }^{11}$ showed that cardiac angiogenesis was involved in the development of adapted right ventricular hypertrophy. Despite right ventricular hypertrophy and angiogenic factors overexpression (eg, HIf- $1 \alpha$ and VEGF), CTEPH animals did not adapt their right ventricle microcirculation as accurately as the shunt animals. Impairment of adaptive angiogenesis leads to imbalance between oxygen consumption and supply, worsening the effect of decreased coronary blood flow. ${ }^{31}$ The mechanism of impairment of angiogenesis and transition to maladaptive right ventricular function is not known. Our hypothesis is that an impairment of angiogenesis due to uncoupling of HiF- $1 \alpha$ and VEGF-A and glycolytic shift of the mitochondrial metabolism led to right ventricular dysfunction. In the CTEPH model, the right ventricle must increase its myocardial mass because the pulmonary pressures rise rapidly. We hypothesized that angiogenesis was not sufficient to supply the increased oxygen demand in the right ventricle, leading to myocardial ischemia and that relative right ventricular ischemia initiates the switch via HIF- $1 \alpha$ signaling. HIF- $1 \alpha$ may increase the activity of pyruvate dehydrogenase kinase, inhibiting the use of pyruvate for adenosine triphosphate production. ${ }^{26}$ This, in turn, increases the rate of glycolysis and decreases production of mitochondrial reactive oxygen species. HIF1- $\alpha$ also stimulates angiogenesis by increasing the expression of
VEGF. Some evidence suggests that an uncoupling of HIF- $1 \alpha$ and VEGF mediated by microRNA-34a may lead to decrease of $\mathrm{CD}$, and thus, increase myocardial hypoxia. ${ }^{32}$

More recently, Wong and colleagues ${ }^{33}$ demonstrated that the right ventricle had less mechanical efficiency and less capacity to use oxygen in advanced-stage disease (New York Heart Association functional class III compared with functional class II). Discrepancy between the $\mathrm{CD}$ and right ventricular SW may help us to understand the inability of the right ventricle under end-stage PH to correctly use oxygen despite the metabolic shift. In addition to myocardial malperfusion, myocardial fibrosis seemed to be associated with worse right ventricular function and maladaptive remodeling. Whether fibrosis is a consequence of chronic ischemia within the myocardium remains unknown. However, we found that $\mathrm{CD}$ and fibrosis were inversely correlated. A major clinical implication of our study is that it offers, in a large animal model, support for therapies targeting neovascularization or angiogenesis. Although HIF- $1 \alpha$ or VEGF upregulation was not sufficient to compensate the right ventricle in the CTEPH model, a higher dose delivered through gene therapy, or CD34-positive cells therapy, could in the future prove helpful. We are at the very early stages of this investigation.

\section{Limitations}

The study has several limitations. First, this study could not provide a mechanistic answer for the difference in right ventricular adaptation between our 2 models of chronic pressure overload. We observed a mismatch between CD and the structural and functional remodeling of the right ventricle in the CTEPH model, but its cause remains unknown. Although we found a strong association between lower CD to SW ratio, fibrosis, and right ventricular maladaptation, we did not perform a recovery study that could demonstrate the causal relationship. We also investigated a panel of key regulating genes but did not analyze a more complex network of regulating factors. The study offers strong base for future work targeting CD in the right heart. Second, chronic pressure overload was induced in growing piglets, a process that could theoretically interfere with mechanisms of right ventricle adaptation. Because of the growth rate of swine $(20 \mathrm{~kg}$ at basal state to $50 \mathrm{~kg}$ after 20 weeks) and because of the duration of the study protocol (20 weeks), we chose to use small piglets at the basal state. We aimed to study right ventricular adaptation in the face of a chronic pressure overload regimen. We know that the CTEPH model is achieved after 6 weeks and Rondelet and colleagues ${ }^{12}$ demonstrated that $\mathrm{PH}$ is obtained in the shunt model after 12 weeks. To reproduce the chronic state of right ventricular pressure overload, we assessed the models after 20 weeks of evolution. Finally, it would be interesting to have more time points in terms of myocardial CD assessment. But this could 
not be possible without scarring the animals and this kind of protocol would require too many animals.

\section{CONCLUSIONS}

The right ventricular adaptative response to CPOS differed between the 2 models. Our results suggest that adaptive right ventricular remodeling to pressure overload is correlated to increased right ventricular $\mathrm{CD}$ and a low rate of myocardial fibrosis. The $\mathrm{CD}$ to $\mathrm{SW}$ ratio could be a promising index to assess right ventricular maladaptation to a pressure overload state. SW could be calculated after right ventricle catheterization. Further studies are needed to explore the correlation between the assessment of the myocardial perfusion with nuclear imaging and the myocardial $\mathrm{CD}$ in the right ventricle. A causality relationship cannot be established with our data and the mechanisms underlying these findings remain unknown. Further studies are needed to elucidate the mechanism of right ventricular microvascularization regulation and to determine a mean to calculate the $\mathrm{CD}$ to $\mathrm{SW}$ ratio applicable to clinical practice.

\section{Conflict of Interest Statement}

Authors have nothing to disclose with regard to commercial support.

\section{References}

1. Humbert M, Sitbon O, Chaouat A, Bertocchi M, Habib G, Gressin V, et al. Survival in patients with idiopathic, familial, and anorexigen-associated pulmonary arterial hypertension in the modern management era. Circulation. 2010;122:156-63.

2. Haddad F, Doyle R, Murphy DJ, Hunt SA. Right ventricular function in cardiovascular disease, part II: pathophysiology, clinical importance, and management of right ventricular failure. Circulation. 2008;117:1717-31.

3. Hopkins WE, Waggoner AD. Severe pulmonary hypertension without right ventricular failure: the unique hearts of patients with Eisenmenger syndrome. Am J Cardiol. 2002;89:34-8.

4. Hopkins WE. The remarkable right ventricle of patients with Eisenmenger syndrome. Coron Artery Dis. 2005;16:19-25.

5. McLaughlin VV, Presberg KW, Doyle RL, Abman SH, McCrory DC, Fortin T, et al. Prognosis of pulmonary arterial hypertension: ACCP evidence-based clinical practice guidelines. Chest. 2004;126:78S-92S.

6. Hopkins WE, Ochoa LL, Richardson GW, Trulock EP. Comparison of the hemodynamics and survival of adults with severe primary pulmonary hypertension or Eisenmenger syndrome. J Heart Lung Transpl. 1996;15:100-5.

7. Bogaard HJ, Natarajan R, Henderson SC, Long CS, Kraskauskas D, Smithson L, et al. Chronic pulmonary artery pressure elevation is insufficient to explain right heart failure. Circulation. 2009;120:1951-60.

8. Sutendra G, Dromparis P, Paulin R, Zervopoulos S, Haromy A, Nagendran J, et al. A metabolic remodeling in right ventricular hypertrophy is associated with decreased angiogenesis and a transition from a compensated to a decompensated state in pulmonary hypertension. J Mol Med (Berl). 2013;91:1315-27.

9. Gomez A, Bialostozky D, Zajarias A, Santos E, Palomar A, Martinez ML, et al. Right ventricular ischemia in patients with primary pulmonary hypertension. J Am Coll Cardiol. 2001;38:1137-42.

10. Izumiya Y, Shiojima I, Sato K, Sawyer DB, Colucci WS, Walsh K. Vascular endothelial growth factor blockade promotes the transition from compensatory cardiac hypertrophy to failure in response to pressure overload. Hypertension. 2006:47:887-93.

11. Sano M, Minamino T, Toko H, Miyauchi H, Orimo M, Qin Y, et al. p53-induced inhibition of Hif-1 causes cardiac dysfunction during pressure overload. Nature. 2007;446:444-8.
12. Rondelet B, Kerbaul F, Motte S, van Beneden R, Remmelink M, Brimioulle S, et al. Bosentan for the prevention of overcirculation-induced experimental pulmonary arterial hypertension. Circulation. 2003;107:1329-35.

13. Mercier O, Tivane A, Dorfmuller P, de Perrot M, Raoux F, Decante B, et al Piglet model of chronic pulmonary hypertension. Pulmon Circ. 2013;3: 908-15.

14. Noly PE, Guihaire J, Coblence M, Dorfmuller P, Fadel E, Mercier O. Chronic thromboembolic pulmonary hypertension and assessment of right ventricular function in the piglet. $J$ Vis Exp. 2015;e53133.

15. Rondelet B, Dewachter C, Kerbaul F, Kang X, Fesler P, Brimioulle S, et al Prolonged overcirculation-induced pulmonary arterial hypertension as a cause of right ventricular failure. Eur Heart J. 2012;33:1017-26.

16. Wilkinson JL. Haemodynamic calculations in the catheter laboratory. Heart. 2001;85:113-20.

17. McMichael J, Sharpey-Schafer EP. Cardiac output in man by a direct Fick method: effects of posture, venous pressure change, atropine, and adrenaline. Br Heart J. 1944;6:33-40.

18. Hoeper MM, Maier R, Tongers J, Niedermeyer J, Hohlfeld JM, Hamm M, et al. Determination of cardiac output by the Fick method, thermodilution, and acetylene rebreathing in pulmonary hypertension. Am J Respir Crit Care Med. 1999; 160:535-41.

19. Burkhoff D, Mirsky I, Suga H. Assessment of systolic and diastolic ventricular properties via pressure-volume analysis: a guide for clinical, translational, and basic researchers. Am J Physiol. 2005;289:H501-12.

20. Pagnamenta A, Dewachter C, McEntee K, Fesler P, Brimioulle S, Naeije R. Early right ventriculo-arterial uncoupling in borderline pulmonary hypertension on experimental heart failure. J Appl Physiol. 2010;109:1080-5.

21. Galie N, Hoeper MM, Humbert M, Torbicki A, Vachiery JL, Barbera JA, et al. Guidelines for the diagnosis and treatment of pulmonary hypertension. Eur Respir J. 2009;34:1219-63.

22. Lang RM, Bierig M, Devereux RB, Flachskampf FA, Foster E, Pellikka PA, et al. Recommendations for chamber quantification: a report from the American Society of Echocardiography's Guidelines and Standards Committee and the Chamber Quantification Writing Group, developed in conjunction with the European Association of Echocardiography, a branch of the European Society of Cardiology. J Am Soc Echocardiogr. 2005;18:1440-63.

23. Hadi AM, Mouchaers KT, Schalij I, Grunberg K, Meijer GA, VonkNoordegraaf A, et al. Rapid quantification of myocardial fibrosis: a new macro-based automated analysis. Cell Oncol (Dordr). 2011;34:343-54.

24. Ryan JJ, Archer SL. The right ventricle in pulmonary arterial hypertension: disorders of metabolism, angiogenesis and adrenergic signaling in right ventricular failure. Circ Res. 2014;115:176-88.

25. Baldwa S, Rana M, Canty JM Jr, Fallavollita JA. Comparison of thallium deposition with segmental perfusion in pigs with chronic hibernating myocardium. Am J Physiol. 2008;295:H2522-9.

26. Rich S. Right ventricular adaptation and maladaptation in chronic pulmonary arterial hypertension. Cardiol Clin. 2012;30:257-69.

27. Drake JI, Bogaard HJ, Mizuno S, Clifton B, Xie B, Gao Y, et al. Molecular signature of a right heart failure program in chronic severe pulmonary hypertension. Am J Respir Cell Mol Biol. 2011;45:1239-47.

28. Greyson CR. Pathophysiology of right ventricular failure. Crit Care Med. 2008; 36:S57-65.

29. Hudlicka O, Brown M, Egginton S. Angiogenesis in skeletal and cardiac muscle Physiol Rev. 1992;72:369-417.

30. Shiojima I, Sato K, Izumiya Y, Schiekofer S, Ito M, Liao R, et al. Disruption of coordinated cardiac hypertrophy and angiogenesis contributes to the transition to heart failure. J Clin Invest. 2005;115:2108-18.

31. Toyota E, Matsunaga T, Chilian WM. Myocardial angiogenesis. Mol Cell Biochem. 2004;264:35-44.

32. van Rooij E, Purcell AL, Levin AA. Developing microRNA therapeutics. Circ Res. 2012;110:496-507.

33. Wong YY, Ruiter G, Lubberink M, Raijmakers PG, Knaapen P, Marcus JT, et al Right ventricular failure in idiopathic pulmonary arterial hypertension is associated with inefficient myocardial oxygen utilization. Circ Heart Fail. 2011;4:700-6.

Key Words: right ventricle, adaptive remodeling, pulmonary hypertension, angiogenesis, fibrosis 